\title{
Cognitive AF Relay Networks over Asymmetric Shadowing/ Fading Channels in the Presence of Low-Rate Feedback
}

\author{
Eylem Erdoğan
}

Department of Electrical and Electronics Engineering, İstanbul Medeniyet University School of Engineering and Natural Science, İstanbul, Turkey

Cite this article as: E. Erdoğan, "Cognitive AF Relay Networks over Asymmetric Shadowing/Fading Channels in the Presence of Low-Rate Feedback", Electrica, vol. 18, no: 2, pp. 172-176, 2018.

\begin{abstract}
This study investigates the performance of a cognitive amplify-and-forward (AF) relay network over composite asymmetric multipath/shadowing fading channels, whereby a new power allocation method is adopted at the secondary source and relay, assuming the mean-value interference channel. To quantify the performance of the proposed structure, closed-form outage probability and ergodic capacity expressions are derived. Moreover, asymptotic ergodic capacity analysis is performed to observe the effects of limited feedback and fading/shadowing severity in the system. The analytical results are validated with Monte-Carlo simulations, and they show that the proposed structure can be a realistic scenario for next-generation wireless systems, and a system designer can test the overall performance of the proposed system without dealing with complex prototypes.
\end{abstract}

Keywords: Cognitive radio, multipath/shadowing fading channels, cooperative communication

\section{Corresponding Author: \\ Eylem Erdoğan}

\section{E-mail:}

eylem.erdogan@medeniyet.edu.tr

Received: 12.12 .2017

Accepted: 25.04 .2018

(c) Copyright 2018 by Electrica

Available online at

http://electrica.istanbul.edu.tr

DOI: 10.26650/electrica.2018.65047

\section{Introduction}

Wireless data traffic has grown tremendously over the past years as the number of users and applications have increased. To meet the data traffic, several promising technologies have been developed. Among them, relay aided communication (also known as cooperative communication) and cognitive radio (CR) have been recognized as two of the most important enablers [1].

Cognitive radio proposes the spectrum-sharing paradigm to enhance the radio frequency spectrum efficiency in wireless systems while keeping the harmful interference between primary and secondary networks at a minimum level [2]. Different techniques have been considered for spectrum sharing so far. Among them, underlay model, which allows the secondary user (SU) to transmit simultaneously with the primary user (PU) in the PU's frequency band, has become a practical solution for next generation wireless systems.

Relay aided transmission on the other side, can provide a reliable communication for the secondary networks by placing intermediate relay nodes between the secondary source and destination. Hence, it can exploit the spatial diversity. In relay-aided communication, amplify and forward (AF) and decode and forward (DF) relaying techniques have been considerably investigated. In DF, the relay detects the received signal and forwards the uncoded signal to the destination. However, in the AF mode, relay simply scales and then transmits the scaled version of the signal [3]. Hence, due to its simple structure, relay aided communication has been adopted into several standards such as 3GPP LTE-Advanced and IEEE 802.16j [4]. In this respect, a wide range of studies in the literature have been considered cognitive cooperative structures (see, e.g., and the references therein) [5-12]. AF method was studied in a CR relay network where outage probability was examined for Rayleigh fading channels [5]. A general case of was considered where both outage and error probability were derived for Nakagami- $m$ fading channels $[5,6]$. Considering DF relaying in Nakagami-m fading, outage probability was examined in and 
the diversity gains of AF and DF CR systems was investigated in $[7,8]$. Moreover, physical layer secrecy performance of CR DF relay network was investigated in [9]-[10]. Yang et al. [11] derived the outage probability performance of dual-hop AF CR network over $\eta-\mu$ fading channels in and Li et al. [12] studied the impact of uncorrelated fading channels on CR relay networks in.

As can be seen above, none of the works above have focused on a cognitive cooperative scheme under practical multipath/ shadowing propagation conditions. Herein, we considered an AF cognitive relaying scheme where the transmission between secondary source $(S)$ and relay $(R)$ is impaired by fading and shadowing effects. To do so, generalized-K composite fading model which can incorporate both fading and shadowing effects is used [13]. Considering that the second hop is modelled with Nakagami-m fading due to asymmetric traffic conditions, we derived closed form expressions of outage probabilitty and ergodic capacity and analyzed the outage probability in the presence of low-rate feedback.

\section{System Model}

This paper considers a cognitive underlay AF relaying scheme in which the communicates with the secondary destination (D) over single relay (R) terminal in the presence of a primary receiver (PR) over asymmetric fading channels. The direct path between $S$ to $D$ is not avaliable as the transmission is performed in a time-division multiple access (TDMA) fashion in two time slots. In the proposed structure, we assume that the $\mathrm{S} \rightarrow \mathrm{R}$ path is detoriarated by the shadowing/fading effects and hence follows the Generalized-K composite fading model. On the other side, $R \rightarrow D$ path is modelled as Nakagami-m fading channel with $\rho_{R, D}$ severity parameter.

In the first hop, both shadowing and fading effects between $\mathrm{S} \rightarrow \mathrm{R}$ path are modeled as i.i.d. Gamma distribution with and fading $\zeta \kappa_{\mathrm{SR}}$ and $\zeta \lambda_{\mathrm{SR}}$ shadowing parameters respectively.

In the first time slot, $\mathrm{S}$ transmits its information to the $\mathrm{R}$. The received signal at the $R$ can be written as

$y_{S R}=P_{S} \kappa_{S R} \lambda_{S R} x_{S}+n_{R}$

where $\kappa_{\mathrm{SR}}$ and $\lambda_{\mathrm{SR}}$ denote the shadowing and channel information respectively and $\chi_{\mathrm{S}}$ is the the source signal. In the second time slot, $R$ transmits the amplified signal to the $D$. The received signal at the $D$ can be written as

$$
y_{R D}=P_{R} h_{R_{D}} G y_{S R}+n_{D}
$$

where $h_{R D}$ denotes the channel information between $R$ and, $D$, $n_{R}$ and $n_{D}$ are the additive white Gaussian noises modeled with zero-mean and unit variance and scaling gain $\mathrm{G}$ is given as
$G=\sqrt{\frac{P_{R}}{P_{S}\left|\kappa_{S R} \lambda_{S R}\right|^{2}+N_{0}}}$.

\section{Mean-Value Power Allocation at Secondary Source and Relay}

In underlay CR networks, it is generally assumed that the PR knows the interference gain of the channel instantaneously. However, this assumption cannot be used in practical wireless networks as it brings huge feedback burden and feedback errors. To reduce the feedback burden and to prevent feedback errors, the practical mean-value (MV) power allocation technique can be used. In MV power allocation, the PR obtains the mean value of the feedback gain and feeds back to the secondary system. Therefore, $\mathrm{S}$ and $\mathrm{R}$ powers can be written as $\mathrm{P}_{\mathrm{S}}$ $\operatorname{Min}\left\{\frac{Q_{p}}{E\left[\left|\kappa_{S P} \lambda_{S P}\right|^{2}\right]}, P_{\max }\right\}$ and $\left\{\frac{Q_{p}}{E\left[\left.h_{R, P}\right|^{2}\right]}, P_{\max }\right\}$, where $\kappa_{\mathrm{SP}}$ and $\lambda_{\mathrm{SP}}$ show the shadowing and channel informations between $S$ and $P R, h_{R, P}$ is the channel information between $R$ and $P R, Q_{p}$ is the maximum tolerable interference power of the PR and $P_{\max }$ shows the maximum power available in the system.

\section{End-to-End Signal to Noise Ratio Calculation}

By substituting (1) and (3) into (2), end-to-end (e2e) SNR can be written as

$\gamma_{e 2 e}=\frac{\gamma_{S R} \gamma_{R D}}{\gamma_{S R}+\gamma_{R D}+1}$

where $\Upsilon_{S R}$ and $\Upsilon_{R D}$ can be written as

$\gamma_{S R}=\min \left(\frac{Q_{p}}{E\left[\left|\kappa_{S P} \lambda_{S P}\right|^{2}\right]}, P_{\max }\right)\left|\kappa_{S R} \lambda_{S R}\right|^{2}$,

$\gamma_{R D}=\min \left(\frac{Q_{p}}{E\left[\left|h_{R P}\right|^{2}\right]}, P_{\max }\right)\left|h_{R D}\right|^{2}$.

As the analysis of (4) is theoretically complicated, obtaining the statistics of SNR can not be possible. However, (4) can be simplified as

$\gamma_{e 2 e} \leq \gamma_{\mathrm{up}}=\min \left(\gamma_{S R}, \gamma_{R D}\right)$.

\section{Performance Analysis}

In this section, outage probability and ergodic capacity expressions are obtained for the proposed structure.

\section{Outage Probability}

As both $\lambda_{\mathrm{SR}}$ and $\kappa_{\mathrm{SR}}$ are independent and Gamma distributed, the $C D F$ of $\Upsilon_{S R}$ can be expressed as

$F_{\gamma_{S R}}(\gamma)=\int_{0}^{\infty} F_{\lambda_{S R}}\left(\frac{\gamma}{x}\right) f_{\kappa_{S R}}(x) d x$ 
With the aid of [14], $\mathrm{F}_{\mathrm{KSR}_{\mathrm{S}}}$ can be given as can be seen in (8) at the top of the next page. In (8), $\Upsilon$ (...) shows the lower incomplete Gamma function [14, eqn. 8.350.1]. The derivative of $\mathrm{F}_{\mathrm{KSR}}(\chi)$ can be obtained as $F_{\lambda_{S R}}\left(\frac{\gamma}{x}\right)$

$$
\begin{aligned}
& F_{\kappa_{S R}}(x)=\frac{\gamma\left(\zeta_{\kappa_{S R}} \frac{\zeta_{\kappa_{S R}} x}{\min \left(\frac{Q_{p}}{E\left[\left|\kappa_{S P} \lambda_{S P}\right|^{2}\right]}, P_{\max }\right)}\right)}{\Gamma\left(\zeta_{\kappa_{S R}}\right)} \\
& =1-e^{-\frac{\zeta_{S R} x}{\min \left(\frac{Q_{p}}{E\left[\left|\kappa_{S P} \lambda_{S P}\right|^{2}\right]^{2}} P_{\max }\right)}} \sum_{v=0}^{\zeta_{S R}-1}\left(\frac{\zeta_{\lambda_{S R}} x}{\min \left(\frac{Q_{p}}{E\left[\left|\kappa_{S P} \lambda_{S P}\right|^{2}\right]}, P_{\max }\right)}\right)^{v} \times \frac{1}{v !} .
\end{aligned}
$$

can be found similarly by changing the variables in (8) as

$f_{\kappa_{S R}}(x)=e^{-\frac{\zeta_{\kappa_{S R}} x}{\min \left(\frac{Q_{p}}{E\left[\left|\kappa_{S P} \lambda_{S P}\right|^{2}\right]}, P_{\max }\right)}}$

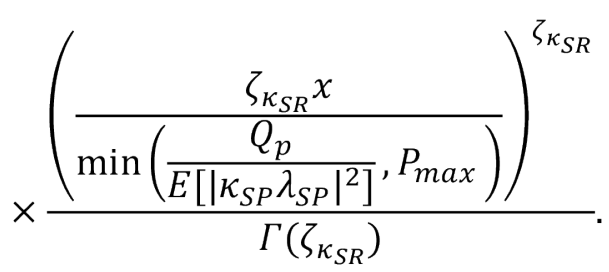

$F_{\lambda_{S R}}\left(\frac{\gamma}{x}\right)$

$=1-e^{-\frac{\zeta_{\lambda_{S R}} \gamma}{x}} \sum_{v=0}^{\zeta_{\lambda_{S R}}-1}\left(\frac{\zeta_{\lambda_{S R}} \gamma}{x}\right)^{v} \frac{1}{v !}$

By substituting (9) and (10) into (7) with the help of [14, eqn. 3.471.9] and after few manipulations, $F_{\Upsilon_{S R}}(Y)$ can be obtained as can be seen in (11) at the top of the page. In (11) $\mathrm{K}_{\mathrm{v}}($.) shows the modified Bessel function of the second kind [15, 51]. On the other hand, the CDF of the $\Upsilon_{\mathrm{RD}}$ can be obtained as

$$
\begin{aligned}
& F_{Y S R}(\gamma)=1-\frac{2}{\Gamma\left(\zeta_{K_{S R}}\right)} \sum_{v=0}^{\zeta_{\lambda_{S R}-1}}\left(\frac{\zeta_{\kappa_{S R}}}{\min \left(\frac{Q_{p}}{E\left[\left|\kappa_{S P} \lambda_{S P}\right|^{2}\right]}, P_{\max }\right)}\right)^{\frac{\zeta_{\kappa_{S}}+v}{2}} 1 / v !
\end{aligned}
$$

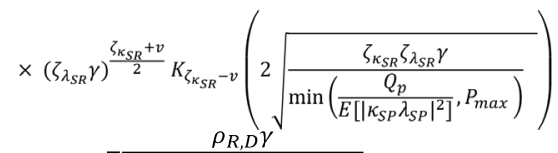

$$
\begin{aligned}
& F_{\gamma_{R, D}}(\gamma)=1-e^{-\frac{\rho_{R, D} \gamma}{\min \left(\frac{Q_{p}}{E\left[\left|h_{R P}\right|^{2}\right]} P_{\max }\right)}} \\
& \times \sum_{v=0}^{\rho_{R, D^{-1}}}\left(\frac{\rho_{R, D} \gamma}{\min \left(\frac{Q_{p}}{E\left[\left|h_{R P}\right|^{2}\right]}, P_{\max }\right)}\right)^{v} \frac{1}{v !},
\end{aligned}
$$

and with the aid of (6), $\mathrm{F}_{\Upsilon_{U P}}(Y)$ can be expresed as

$F_{\gamma_{u p}}(\gamma)=1-\left(1-F_{\gamma_{S, R}}(\gamma)\left(1-F_{\gamma_{R, D}}(\gamma)\right)\right.$

Outage probability is defined as the probability of SNR falling below a predefined threshold, $\Upsilon_{\text {th }}$ and can be expressed as $\mathrm{P}_{\text {out }}=\mathrm{F}_{\Upsilon_{\mathrm{UP}}}\left(\mathrm{Y}_{\text {th }}\right)$. It can be obtained easily by substituting (11) and (12) into (13).

\section{Ergodic Capacity}

Ergodic capacity is one of the most important performance indicators in wireless systems. Ergodic capacity is the maximum achievable rate that a communication system can obtain. Mathematically speaking, it can be expressed as where E [.] denotes the expectation operation. To the best of our knowledge, there is no direct

$C_{E}=\frac{1}{2} E\left[\log _{2}\left(1+\gamma_{e 2 e}\right)\right]$

method to find the closed form solution of ergodic capacity. However, a tight upper bound can be obtained by adopting yup to the above expression as

$C_{E} \leq \frac{1}{2} E\left[\log _{2}\left(1+\gamma_{u p}\right)\right]$
$=\frac{\log _{2}(e)}{2} \int_{0}^{\infty} \frac{1}{1+\gamma} \breve{F}_{\text {up }}(\gamma) d \gamma$

where the simple form of $F_{\Upsilon_{\text {up }}}(\Upsilon), \breve{F}_{\Upsilon_{\text {up }}}(\Upsilon)$ denotes the complementary $C D F$ of $\Upsilon_{\text {up }}$ i.e., $\breve{F}_{\Upsilon_{u p}}(\Upsilon)=1-F_{\Upsilon_{u p}}(\Upsilon)$. By subsituting $F_{\Upsilon_{u p}}(\Upsilon)$ into (15) and after few manipulations, $C_{E}$ can be found as given in (16) at the top of the next page. In (16), Monte-Carlo simulations show that $\mathrm{I}_{3}$ can be negligible at medium and high SNRs as it is the multiplication of two CDF expressions. $\mathrm{I}_{2}$, can be obtained by using [14, eqn. 3.383.10] as can be seen in (17). Note that, $\Gamma$ (...) denotes the upper incomplete Gamma function [14, eqn. 8.350.2]. and $\Gamma$ (.) stands for the Gamma function [14, eqn. 8.339.1]. $\mathrm{I}_{1}$ on the other hand can be derived in closed form by using well known software programs such as MAPLE or MATHEMATICA as can be seen in (18). Note that, $\varnothing_{1}, \varnothing_{2}, \varnothing_{3}$ and $\varnothing_{4}$ can be obtained as given in (19-22).

$$
\begin{aligned}
& C_{E} \leq \frac{\log _{2}(e)}{2}(\underbrace{\int_{0}^{\infty} \frac{1}{1+\gamma} \breve{F}_{S R}(\gamma) d \gamma}_{I_{1}}+\underbrace{\int_{0}^{\infty} \frac{1}{1+\gamma} \breve{F}_{\gamma D}(\gamma) d \gamma}_{I_{2}}-\underbrace{\int_{0}^{\infty} \frac{1}{1+\gamma} \breve{F}_{S R}(\gamma) \breve{F}_{Y_{R D}}(\gamma) d \gamma}_{I_{3}}) .
\end{aligned}
$$

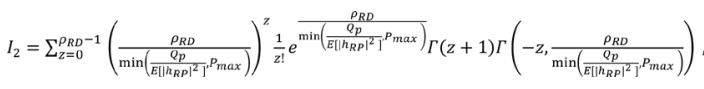

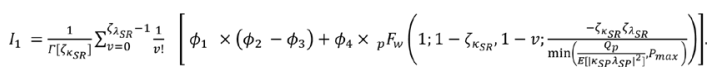

$$
\begin{aligned}
& \phi_{1}=\left(\frac{\zeta_{\kappa_{S R}} \zeta_{\lambda_{S R}}}{\min \left(\frac{Q_{p}}{E\left[\left|\kappa_{S P} \lambda_{S P}\right|^{2}\right]}, P_{\max }\right)}\right)^{\frac{\zeta_{\kappa_{S R}}+v}{2}} \\
& \times \pi^{2} \csc \left[\pi\left(\zeta_{\kappa_{S R}}-v\right]\right. \text {. }
\end{aligned}
$$


$\phi_{2}=J_{-\zeta_{\kappa_{S R}}+v}\left(2 \sqrt{\frac{\zeta_{\kappa_{S R}} \zeta_{\lambda_{S R}}}{\min \left(\frac{Q_{p}}{E\left[\left|\kappa_{S P} \lambda_{S P}\right|^{2}\right]}, P_{\max }\right)}}\right) \times \csc [v \pi]$

$\phi_{3}=J_{\zeta_{\kappa_{S R}}-v}\left(2 \sqrt{\frac{\zeta_{\kappa_{S R}} \zeta_{\lambda_{S R}}}{\min \left(\frac{Q_{p}}{E\left[\left|\kappa_{S P} \lambda_{S P}\right|^{2}\right]}, P_{\max }\right)}}\right) \times \csc \left[\zeta_{\kappa_{S R}} \pi\right]$,

$\phi_{4}=\Gamma(v) \Gamma\left(\zeta_{\kappa_{S R}}\right) \times\left(\frac{\zeta_{\kappa_{S R}} \zeta_{\lambda_{S R}}}{\min \left(\frac{Q_{p}}{E\left[\left|\kappa_{S P} \lambda_{S P}\right|^{2}\right]}, P_{\max }\right)}\right)^{\frac{-\zeta_{\kappa_{S R}-v}}{2}}$.

In (18), pFq(.) denotes the generalized hypergeometric function and in $(20,21)$, J.(.) stands for the modified Bessel function of the first kind [15]. By substituting (18) and (17) into (16), ergodic capacity can be obtained as $C_{E} \leq \frac{\log _{2}(e)}{2}\left(I_{1}+I_{2}\right)$.

Moreover, a tight asymptotic bound on the $\mathrm{C}_{\mathrm{E}}$ can be obtained (23) by using Jensen's inequality as

$\underbrace{\frac{1}{2} E\left[\log _{2}\left(1+\gamma_{e 2 e}\right)\right]}_{C_{E}} \leq \underbrace{\frac{1}{2} \log _{2}\left(E\left[1+\gamma_{e 2 e}\right]\right)}_{C_{E}^{\infty}}$.

After several manipulations, $\mathrm{C}_{\mathrm{E}}^{\infty}$ can be obtained as given at the top of the next page in (24).

$C_{E}^{\infty}=\frac{1}{2} \log _{2}\left(\left[1+\frac{\min \left(\frac{Q_{p}}{E\left[\left|\kappa_{S P} \lambda_{S P}\right|^{2}\right]^{2}}, P_{\max }\right) E\left[\left|\kappa_{S P} \lambda_{S P}\right|^{2}\right] \min \left(\frac{Q_{p}}{E\left[\left|h_{R P}\right|^{2}\right]^{2}}, P_{\max }\right) E\left[\left|h_{R D}\right|^{2}\right]}{\min \left(\frac{Q_{p}}{E\left[\left.\left|\kappa_{S P} \lambda_{S P}\right|^{2}\right|^{2},\right.}, P_{\max }\right) E\left[\left|\kappa_{S P} \lambda_{S P}\right|^{2}\right]+\min \left(\frac{Q_{p}}{E\left[\left|h_{R P}\right|^{2}\right]^{2}}, P_{\max }\right) E\left[\left|h_{R D}\right|^{2}\right]}\right]\right)$

\section{Impact of Limited Feedback}

In wireless systems, feedback link is always assumed as full-rated. However, if a network suffers from power constraints, limited feedback occurs. Herein, we assume that the $R \rightarrow D$ path is modelled with Rayleigh distribution (special case of Nakagami- $m$ when $m=1$ ) and the feedback path between $R$ to $D$ is limited. Therefore, the PDF of $\Upsilon_{\mathrm{RD}}$ can be expressed as [16] where $\in$ shows the rate of feedback, i.e. $\in=0$ denotes the full rate feedback. $F_{-}\left(\gamma_{-}(R, D)\right) \wedge$ Lim $(\gamma)$ can be obtained by integrating (25) with respect to $\gamma$ as

$$
\begin{aligned}
& f_{\gamma_{R, D}}^{\operatorname{Lim}}(\gamma)=\frac{e^{-\frac{\gamma}{(1-\epsilon) \min \left(\frac{Q_{p}}{E\left[\left|\kappa_{S P} \lambda_{S P}\right|^{2}\right]^{\prime}} P_{\max }\right)}}}{(1-\epsilon) \min \left(\frac{Q_{p}}{E\left[\left|\kappa_{S P} \lambda_{S P}\right|^{2}\right]}, P_{\max }\right)} \\
& F_{\gamma_{R, D}}^{\operatorname{Lim}}(\gamma)=1-e^{-\frac{\gamma}{(1-\epsilon) \min \left(\frac{Q_{p}}{E\left[\left|\kappa_{S P} \lambda_{S P}\right|^{2}\right]^{3}} P_{\max }\right)} .}
\end{aligned}
$$

By substituting (26) into (13) and by adopting the theoretical steps described above, both outage probability and ergodic capacity can be obtained.

\section{Numerical Results}

In this section, Monte-Carlo simulations are carried out to demonstrate the impact of asymmetric fading/shadowing channels in cognitive AF relaying scheme and the theoretical results are validated with Monte-Carlo simulations. Moreover, for analytical brevity, $\sigma_{\kappa_{S R}}^{2}=\sigma_{\kappa_{S P}}^{2}=\sigma_{\kappa_{S}}^{2}, \sigma_{\lambda_{S R}}^{2}=\sigma_{\lambda_{S P}}^{2}=\sigma_{\lambda_{S}}^{2}$ and Figure 1 illustrates the outage probability performance of the considered scheme. As can be seen, there is almost 10 $\mathrm{dB}$ difference between intense fading/shadowing environment i.e., $\sigma_{\kappa_{S}}^{2}=\sigma_{\lambda_{S}}^{2}=\sigma_{h_{R}}^{2}=1$ and light fading/shadowing i.e., $\sigma_{\kappa_{S}}^{2}=\sigma_{\lambda_{S}}^{2}=\sigma_{h_{R}}^{2}=4$ at $5 \times 10-1 \mathrm{~dB}$. Moreover, after $24 \mathrm{~dB}$, there is no performance gain and the diversity goes to 0 as $P_{S}$ and $P_{R}$ reaches to $P_{\max }$.

Figure 2 depicts the ergodic capacity performance of the proposed scheme. The dash/dot curves (theoretical and asymptotic results) show good agreement with the marker symbols of the Monte- Carlo simulations. It is observed that, there is almost $4 \mathrm{~dB}$ difference between intense and light fading/shadowing environments at 4 bits/channels use.

Figure 3 shows the impact of limited feedback on the performance of ergodic capacity. As seen from the figure, there is almost $10 \mathrm{~dB}$ difference between full-rated feedback ( $\alpha=0$ ) and very limited feedback $(\alpha=0.9)$. Therefore, we can infer that low-rate feedback can degrade the performance of wireless systems.

\section{Conclusion}

In this paper, we propose and analyze a cognitive AF relaying scheme in the presence of intense and light fading/shadowing environments. We believe that the proposed model can be a reliable scheme for next generation wireless systems and a system designer can have a quick idea about the system performance with the help of the theoretical results, without dealing with complex prototypes.

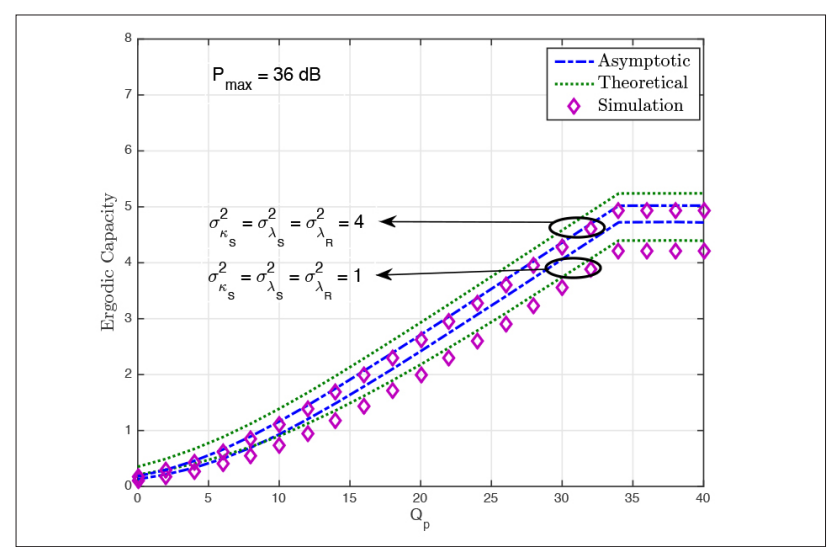

Figure 1. Closed form and theoretical outage probability for different fading/shadowing severity parameters. 


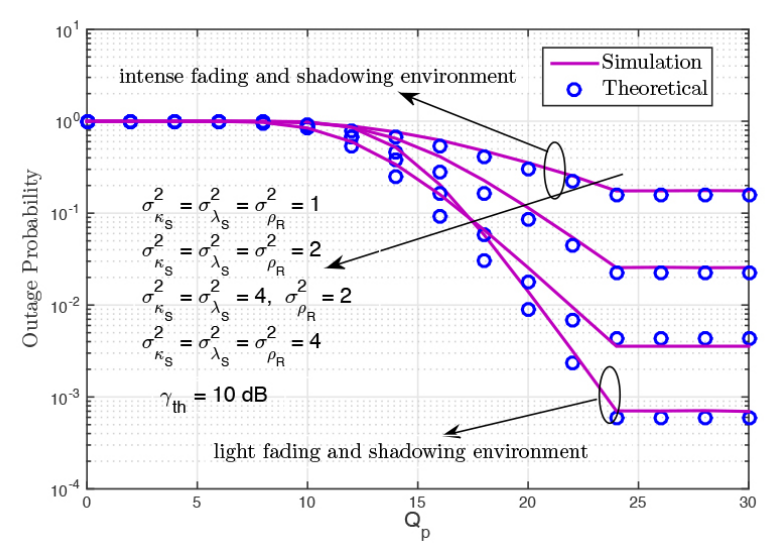

Figure 2. Closed form, theoretical and asymptotic ergodic capacity performance.

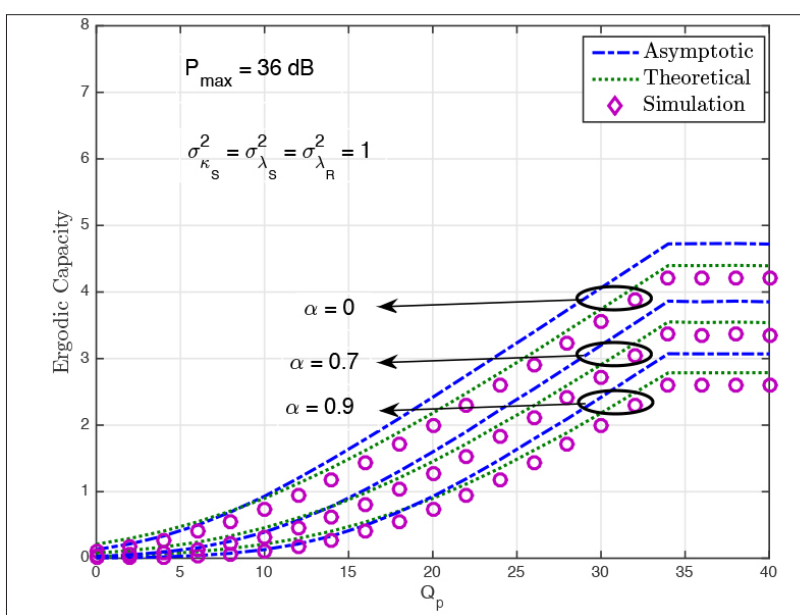

Figure 3. Impact of limited feedback on the performance of the proposed scenario.

Peer-review: Externally peer-reviewed.

Conflict of Interest: The authors have no conflicts of interest to declare.

Financial Disclosure: This study was supported by the Research Fund of Istanbul Medeniyet University, Project Number: F-GAP-2017-1056.

\section{References}

1. C. X. Wang, F. Haider, X. Gao, X. H. You, Y. Yang, D. Yuan, H. M. Aggoune, Harald Haas, s. Fletcher, "Cellular architecture and key technologies for 5G Wireless Communication Networks", IEEE Commun Mag, vol. 52, no. 2, pp. 122-130, 2014.

2. A. Goldsmith, S.A. Jaffar, I. Maric, S. Srinivasa "Breaking spectrum gridlock with cognitive radios: An information theoretic perspective", Proceedings of the IEEE, vol. 97, no. 5, pp. 894-914, 2014.

3. X. Tao, X. Xu, Q. Cui, "An Overview of Cooperative Communications", IEEE Commun Mag, vol. 50, no. 6, pp. 65-71, 2012.

4. E. Dahlman, S. Parkvall, J. Skold, P. Beming, "3G Evolution: HSPA and LTE for Mobile Broadband", New York, Academic Press, 2010.

5. T. Q. Duong, V. N. Q. Bao, H.-J. Zepernick, "Exact outage probability of cognitive AF relaying with underlay spectrum sharing", Electron Lett, vol. 47, no. 17, pp. 1001-1002, 2011.

6. T. Q. Duong, D. B. da Costa, M. Elkashlan, V. N. Q. Bao, “Cognitive amplify-and-forward relay networks over Nakagami-m fading", IEEE Trans Veh Technol, vol. 61, no. 5, pp. 2368-2374, 2012.

7. C. Zhong, T. Ratnarajah, K.K. Wong, "Outage analysis of decode-and-forward cognitive dual-hop systems with the interference constraint in Nakagami-m fading channels", IEEE Trans Veh Technol, vol. 60, no. 6, pp. 2875-2879, 2011.

8. H. Ding, J. Ge, D.B. da Costa, Z. Jiang, "Asymptotic analysis of cooperative diversity systems with relay selection in a spectrum sharing scenario", IEEE Trans Veh Technol, vol. 60, no. 2, pp. 457-472, 2011.

9. R. Zhao, Y. Yuan, L. Fan, Y.C. He, "Secrecy performance analysis of cognitive decode-and-forward relay networks in Nakagami-m fading channels", IEEE Trans Veh Technol, vol. 65, no. 2, pp. 547-563, 2017.

10. D. Chen, Y. Chang and W. Yang, "Physical layer security in cognitive untrusted relay networks", IEEE Access, vol. 6, pp. 7055-7065, Oct. 2017.

11. J. Yang, L. Chen, X. Lei, K. Peppas, T.Q. Duong, "Dual-hop cognitive amplify-and-forward relaying networks over $\eta-\mu$ fading channels", IEEE Trans Veh Technol, vol. 65, no. 8, pp. 6290-6300, 2016.

12. M. Li, H. Yin, Y. Huang, Y. Wang, "Impact of correlated fading channels on cognitive relay networks with generalized relay selection", IEEE Access, vol. 6, pp: 6040-6047, 2017.

13. P.S. Bithas, N.C. Sagias, P.T. Mathiaopoulos, G.K. Karagiannidis, A. A. Rontogiannis, "On the performance analysis of digital communications over generalized-K fading channels", IEEE Commun Lett, vol. 10, no. 5, pp. 353-355, 2006.

14. I. S. Gradshteyn, I.M. Ryzhik, "Table of integrals, series and products," $7^{\text {th }}$ ed. New York: Academic Press, 2007.

15. K.B. Oldham, J. Myland, J. Spanier, "An atlas of functions", $2^{\text {nd }}$ ed. Berlin, Germany: Springer-Verlag, 2008.

16. Y. Ma, D. Zhang, A. Leith, Z. Wang, "Error performance of transmit beamforming with delayed and limited feedback.", IEEE Trans. Wireless Commun, vol. 8, no. 3, pp: 1164-1170, 2009.

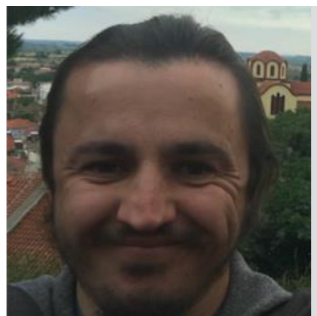

Eylem Erdoğan received B.Sc. and M.Sc. degree from Işıı University, Istanbul, Turkey in 2003 and 2006 and the Ph.D. degree from Kadir Has University, İstanbul, Turkey, all in electronics engineering. He is currently an Assistant Professor in the Department of Electrical and Electronics Engineering, İstanbul Medeniyet University. He was a Post-Doctoral Fellow in Electrical Engineering Department, Lakehead University, Thunder Bay, ON, Canada from 2015 to 2016. He has authored or coauthored around 20 papers in peer reviewed journals and international conferences. His research interests are in the broad areas of wireless communications, including signal processing for wireless communications, the performance analysis of cooperative relaying in cognitive radio networks, mmWave communications and optical frequency bands. 